\title{
A SIMPLE METHOD FOR SIMULATION OF COHERENT SYNCHROTRON RADIATION IN A TRACKING CODE*
}

\author{
M. Borland, ANL, Argonne, IL 60439, USA
}

\begin{abstract}
Coherent synchrotron radiation (CSR) is of great interest to those designing accelerators as drivers for free-electron lasers (FELs). Although experimental evidence is incomplete, CSR is predicted to have potentially severe effects on the emittance of high-brightness electron beams. The performance of an FEL depends critically on the emittance, current, and energy spread of the beam. Attempts to increase the current through magnetic bunch compression can lead to increased emittance and energy spread due to CSR in the dipoles of such a compressor. The code elegant [1] was used for design and simulation of the bunch compressor [2] for the Low-Energy Undulator Test Line (LEUTL) FEL [3] at the Advanced Photon Source (APS). In order to facilitate this design, a fast algorithm was developed based on the 1-D formalism of Saldin and coworkers [4]. In addition, a plausible method of including CSR effects in drift spaces following the chicane magnets was developed and implemented. The algorithm is fast enough to permit running hundreds of tolerance simulations including CSR for 50 thousand particles. This article describes the details of the implementation and shows results for the APS bunch compressor.
\end{abstract}

\section{INTRODUCTION}

It has long been known that electrons traveling through a bending magnet emit radiation. Only recently it has become widely accepted that, when very short electron bunches travel through a dipole magnet, they can emit coherently at wavelengths that are comparable to the bunch length and can propagate in the vacuum chamber. A number of authors (e.g., [5]) have delved in detail into the effects of CSR on the electron beam. Here, I describe in simple terms why CSR has an impact on the electron beam.

Because electrons traverse the dipole on an arc of a circle, radiation from one part of the arc can catch up with electrons on another part of the arc. This, together with the fact that the emission is coherent and at a wavelength comparable to the bunch length, results in a modulation of the energy along the bunch. This is similar to a wakefield, but, unlike wakefields, CSR affects the particles ahead of the emitting particles rather than behind. (For convenience, I'll use the phrase "CSR wake" in spite of this difference.)

Since this is happening inside a dipole magnet, the energy modulation results in a modulation of the transverse slopes in the bending plane, which increases the projected transverse emittance in the bending plane. The mechanism

\footnotetext{
* Work supported by the U.S. Department of Energy, Office of Basic Energy Sciences, under Contract No. W-31-109-ENG-38.
}

behind the emittance growth per se is the same as that for emittance growth due to quantum excitation. An important difference is that quantum excitation is random and hence increases the slice emittance, as well as the projected emittance.

The CSR model used by elegant is based on an equation [4] for the energy change of an arbitrary line-charge distribution as a function of the position in the bunch and in a dipole magnet. Effects of changes in the longitudinal distribution within a dipole are included. However, because Saldin's result is for a line-charge distribution, this model does not include the effect of the transverse distribution of the beam on the CSR nor does it include the variation of the CSR across the beam. The model does include the effect of the transverse beam distribution on the amount of emittance growth due to the energy modulation.

CSR in drift spaces is included by propagating the terminal CSR wake in each bend through the drifts with the beam. The method used is believed to be plausible but has not been derived in a rigorous fashion. Included is a plausible method for modifying the intensity of the CSR as it travels with the beam.

\section{SIMULATION OF CSR EFFECTS IN DIPOLES}

\subsection{Theoretical Basis}

I begin this section with a recapitulation of the relevant results from Saldin et al. The rate of change of energy in the bunch is given by

$$
\frac{d E(s, \phi)}{c d t}=T_{1}(s, R, \phi)+T_{2}(s, R, \phi),
$$

where $R$ is the bend radius, $\phi$ is the angle into the bend (i.e., the average electron position), $s$ is the position within the bunch, and $t$ is time. $T_{1}$ is responsible for most of the CSR effect and transitions into the steady-state result. It is given by

$$
T_{1}=K \int_{s-s_{L}}^{s} \frac{d \lambda}{d z}\left(\frac{1}{s-z}\right)^{\frac{1}{3}} d z
$$

where $\lambda(z)$ is the linear charge density, $s_{L}=\frac{R \phi^{3}}{24}$ is the slippage length, and $K=\frac{-2 e^{2}}{\left(3 R^{2}\right)^{\frac{1}{3}}}$.

The other term, $T_{2}$, is an entrance transient that eventually dies out in a sufficiently long dipole. It is given by

$$
T_{2}=K \frac{\lambda\left(s-s_{L}\right)-\lambda\left(s-4 s_{L}\right)}{s_{L}^{\frac{1}{3}}}
$$



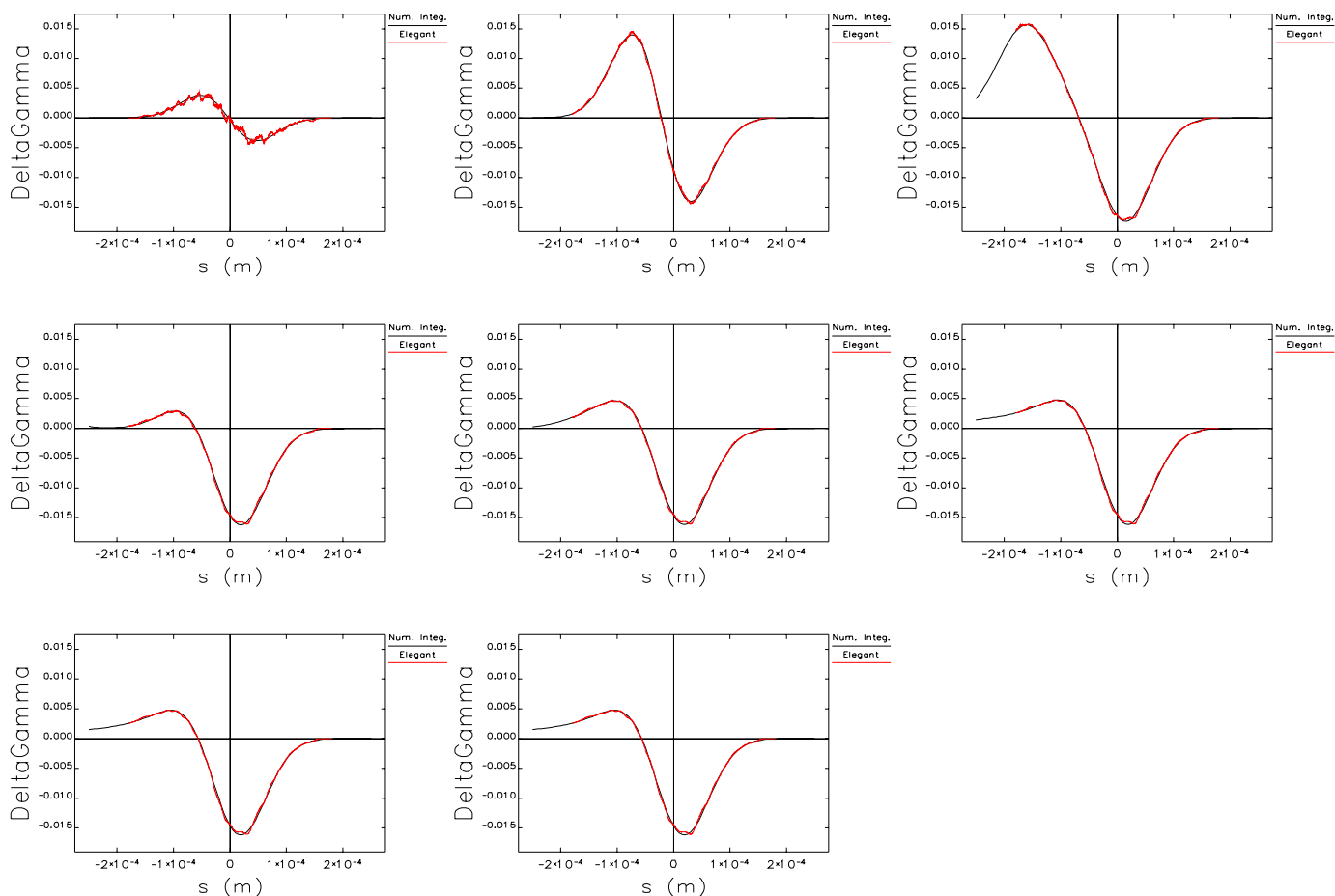

Figure 1: Comparison of elegant simulation and numerical integration, showing the change in $\gamma$ in successive pieces of a dipole. Successive frames are separated by an angle of $20 \mathrm{mrad}$.

To facilitate testing, elegant supports a "steady-state" mode in which, effectively, $s_{L} \rightarrow \infty$. This means that $T_{2} \rightarrow 0$ and the lower limit on the integral for $T_{1}$ goes to $-\infty$.

\subsection{Implementation}

The implementation of these results in elegant relies on splitting each dipole into a user-specified number of pieces. Tests show that 60 pieces is adequate, though typically 100 are used. For each piece, the following steps are peformed:

1. Propagate the entire beam through the piece using a second- or fourth-order canonical integrator [6]. The order is user selectable, but fourth-order is preferred.

2. Compute the CSR wake.

\section{Apply the CSR energy kicks.}

Steps 2 and 3 are detailed below. A more accurate implementation would include the CSR energy kicks in the canonical integration. However, this would have complicated the code and is probably unnecessary in any case. Because the canonical integration through the dipole fields includes the energy dependence of the transport to all orders, the dispersive effects on the transverse coordinates of the particles are computed automatically.

Computation of the CSR wake is performed as follows:

1. Particle arrival times at the end of the dipole piece are binned.
2. This density histogram is smoothed using FFT convolution with a Savitzky-Golay filter [7].

3. The same filter is used to take the derivative of the smoothed density distribution.

4. The $T_{1}$ and $T_{2}$ functions are computed for each bin. Simple sums are used for the integral required by $T_{1}$.

5. Each particle's energy is changed by $\frac{d E}{c d t} \Delta s$ for the bin it occupies, where $\Delta s$ is the central path length of the dipole piece.

One difficulty in these computations comes from noise in the linear density histogram due to use of a finite number of particles and a large number of bins. This is a particular problem when taking the derivative of $\lambda$. Smoothing is used to overcome this problem, at the expense of some loss of information (e.g., real spikes in the current will be reduced). Tests show that using at least 40,000 particles and 3072 bins is required. A first-order Savitzky-Golay filter is used with a half-width of 50 bins.

\subsection{Tests of the Implementation}

Two types of tests were performed to validate the implementation of this algorithm. Both tests made use of a gaussian input beam with 100,000 particles, charge of $1 \mathrm{nC}$, and an rms bunch length of $50 \mu \mathrm{m}$. The bending radius was 1.5 $\mathrm{m}$. This matches the parameters used in the example in Saldin et al. 


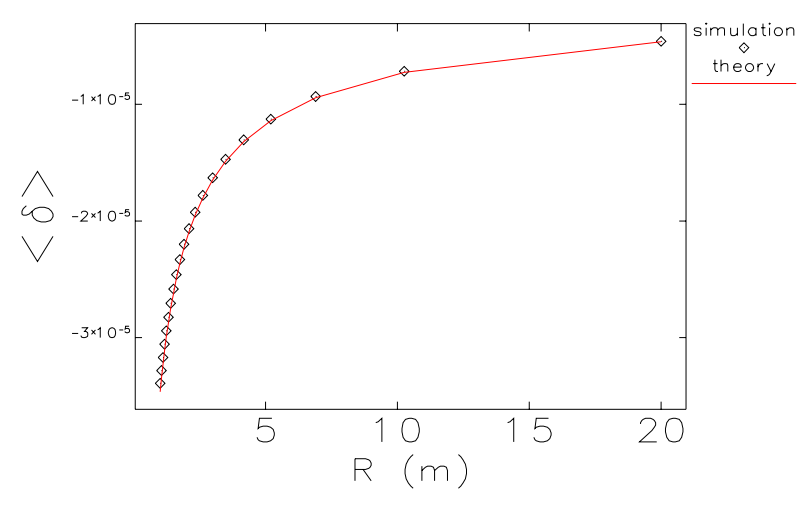

Figure 2: Mean fractional momentum offset vs bending magnet radius.

First, I compared the $\frac{d E}{c d t}$ functions computed by elegant and by numerical integration. This comparison shows excellent agreement, as seen in Figure 1. Differences appear to be due to deviation of the distribution from gaussian in elegant, which hints at the importance of using a detailed beam distribution in the computations, rather than analytical results based on an assumption of a gaussian line density.

The second test made use of the steady-state CSR mode in elegant, allowing comparison with analytical calculations for gaussian bunches. From results given by Saldin et al., one can deduce the mean energy offset and the standard deviation of the energy offset. Letting $\delta=\left(p-p_{o}\right) / p_{o}$, where $p_{o}$ is the central momentum, one obtains

$$
\langle\delta\rangle=-0.3505 \frac{r_{e} Q L_{b}}{e \gamma\left(R^{2} \sigma_{s}^{4}\right)^{\frac{1}{3}}}
$$

and

$$
(\delta)_{S t . D e v}=0.2459 \frac{r_{e} Q L_{b}}{e \gamma\left(R^{2} \sigma_{s}^{4}\right)^{\frac{1}{3}}},
$$

where $L_{b}$ is the length of the dipole and $\sigma_{s}$ is the rms bunch length. elegant reproduces these results very accurately, as seen in Figures 2 through 5.

\section{SIMULATION OF CSR IN DRIFT SPACES}

Simulation of CSR in drift spaces is as important as simulation in dipoles, yet has garnered less attention in the literature. The method I've used is not rigorous but seems plausible. It starts with the assumption that the terminal CSR wake from the dipole propagates with the beam through the elements following the dipole. I assume that the wake does not change shape and that it moves at the speed of light, while the beam moves at a lesser speed. Because it is implausible to imagine that the effect of the intensity of the wake will be unchanging with distance, the intensity is allowed to vary according to one of several models.

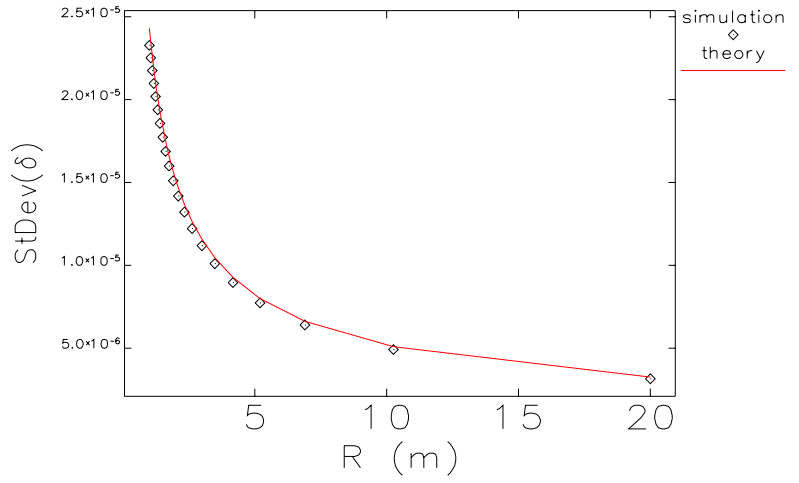

Figure 3: Standard deviation of fractional momentum offset vs bending magnet radius.

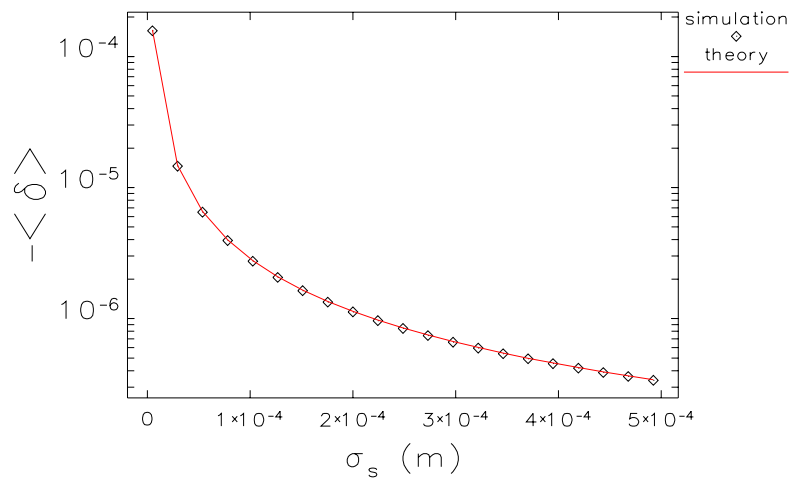

Figure 4: Mean fractional momentum offset vs rms bunch length.

In practice, drifts following dipoles are split into pieces of about $1 \mathrm{~cm}$ length in order to integrate the effect of CSR. If nondrift elements (e.g., quadrupoles) are in the beamline, the effect of CSR in those elements is deferred until the beginning of the next drift space. This was done to simplify the implementation.

elegant supports two methods for modifying the intensity of the wake. One is a simple exponential decay, using a decay parameter supplied by the user. The second is based on assumptions about the opening angle of the radiation. The opening angle of synchrotron radiation for a single electron is given approximately by [8]

$$
\sigma_{\text {rad }}^{\prime}=\frac{0.00055}{E\left(\frac{\lambda_{c}}{\lambda}\right)^{\frac{1}{3}}},
$$

where $E$ is the energy in $\mathrm{MeV}$ and $\lambda_{c}$ is the critical wavelength. For the radiation wavelength, $\lambda$, I use the half-width of the interval containing the central $68 \%$ of the particles. For a gaussian distribution, this is simply the rms bunch length.

Given the opening angle of the single-electron radiation, the rms width of the radiation can be computed as a func- 


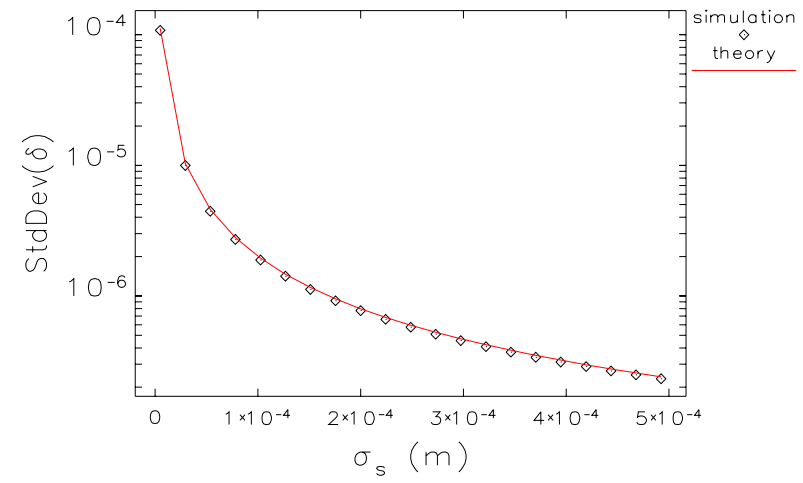

Figure 5: Standard deviation of fractional momentum offset vs rms bunch length.

tion of distance from the source. One obtains

$$
\sigma_{\text {rad }}=\sqrt{\Sigma_{11}+2 \Sigma_{12} z+\left(\Sigma_{22}+\left(\sigma_{\text {rad }}^{\prime}\right)^{2}\right) z^{2}}
$$

where $\Sigma$ is the electron beam sigma matrix and $\mathrm{z}$ is the distance from the end of the dipole. The "intensity reduction factor" is simply

$$
\frac{\sigma_{\text {rad }}(z=0)}{\sigma_{\text {rad }}}=\frac{\sqrt{\Sigma_{11}}}{\sqrt{\Sigma_{11}+2 \Sigma_{12} z+\left(\Sigma_{22}+\left(\sigma_{r a d}^{\prime}\right)^{2}\right) z^{2}}} .
$$

In summary, in the simple model proposed here, the CSR wake changes in intensity when propagating through a drift space by the ratio of the initial radiation beam size to the radiation beam size at each point in the drift space. Note that, if $\Sigma_{12}$ is sufficiently large and negative, the photon beam will converge and the CSR wake will increase in intensity up to some point.

\section{APPLICATION TO THE APS BUNCH COMPRESSOR}

elegant was used to design and explore the performance of the APS bunch compressor [2], which features a fourmagnet chicane with movable dipoles. The bunch at the entrance to the chicane has a nearly gaussian longitudinal distribution but is clearly nongaussian at the entrance to the third dipole. Hence, elegant's use of the actual beam distribution in computing the CSR wake is expected to be important here. Indeed, as Figures 6 through 8 show, the shape of the CSR wake is dramatically different from what would be expected from a gaussian bunch. This is partly due to the appearance of a high-current spike at the head of the bunch. In this example, a $0.4 \mathrm{nC}$ bunch is compressed to yield an average current of about 600A, which is typical of what we plan to run at the APS facility in the near future.

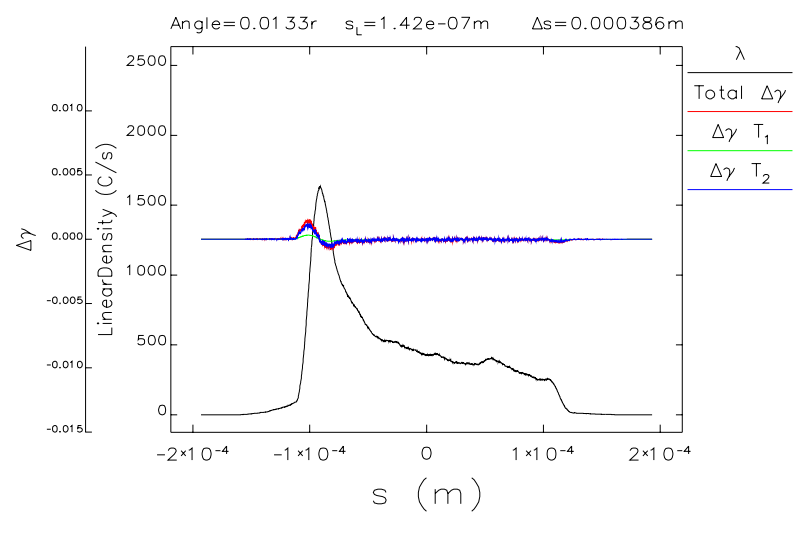

Figure 6: Bunch distribution and CSR wakes near the beginning of the fourth dipole.

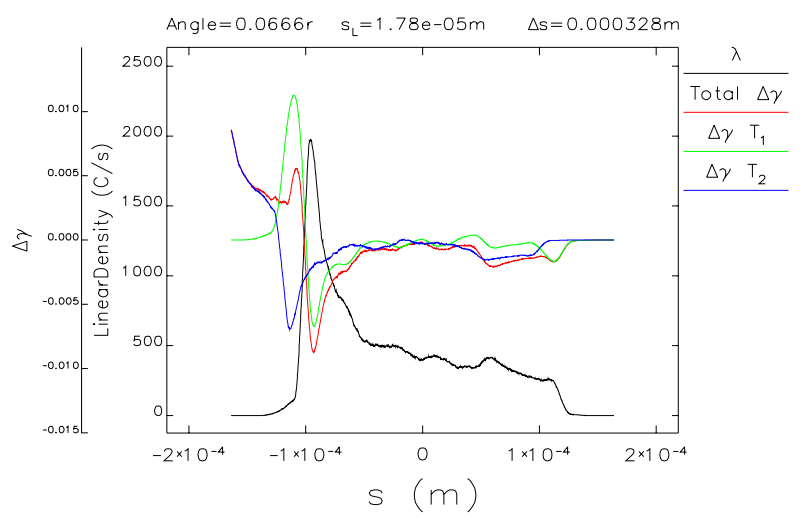

Figure 7: Bunch distribution and CSR wakes at the center of fourth dipole.

\section{REFERENCES}

[1] M. Borland, unpulished program. Manual is on-line at www.aps.anl.gov/asd/oag/manuals/elegant_ver14.1/ elegant.html .

[2] M. Borland, "Design and Performance Simulations of the Bunch Compressor for the APS LEUTL FEL," Proceedings of LINAC 2000, Monterey, California, to be published.

[3] S.V. Milton et al., "Observation of Self-Amplified Spontaneous Emission and Exponential Growth at 530 nm," Phys. Rev. Lett., to be published.

[4] E. L. Saldin, E. A. Schneidmillter, and M. V. Yurkov, "On the coherent radiation of an electron bunch moving in an arc of a circle," NIM A 398 (1997) 392.

[5] B. E. Carlsten and T. O. Raubenheimer, "Emittance growth of bunched beams in bends," Phys. Rev. E, 51, 2, (1995).

[6] E. Forest, "Canonical Integrators as Tracking Codes," in Physics of Particle Accelerators, AIP Conference Proceedings 184.

[7] W. Press, S. Teukolsky, W. Vetterling, and B. Flannery, Numerical Recipes in $C$, 2nd Edition, Cambridge University Press, 1998, p. 650. 


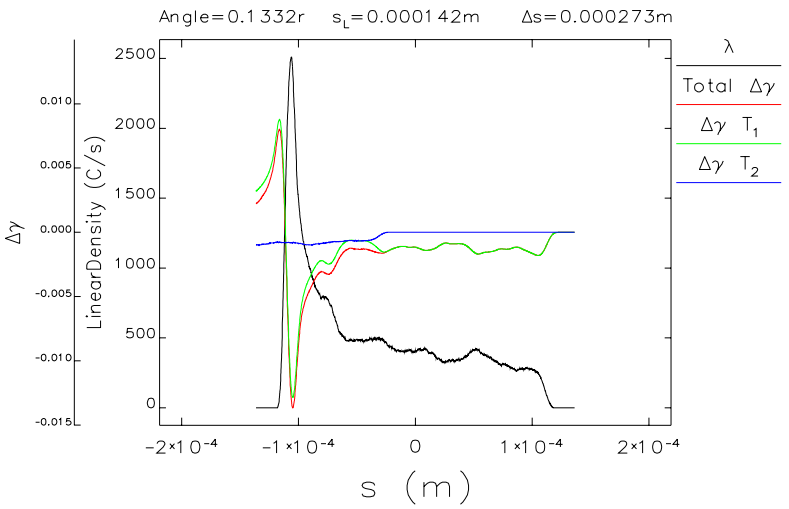

Figure 8: Bunch distribution and CSR wakes at the end of the fourth dipole.

[8] H. Wiedemann, Particle Accelerator Physics, section 9.6.1, Spring-Verlag, . 